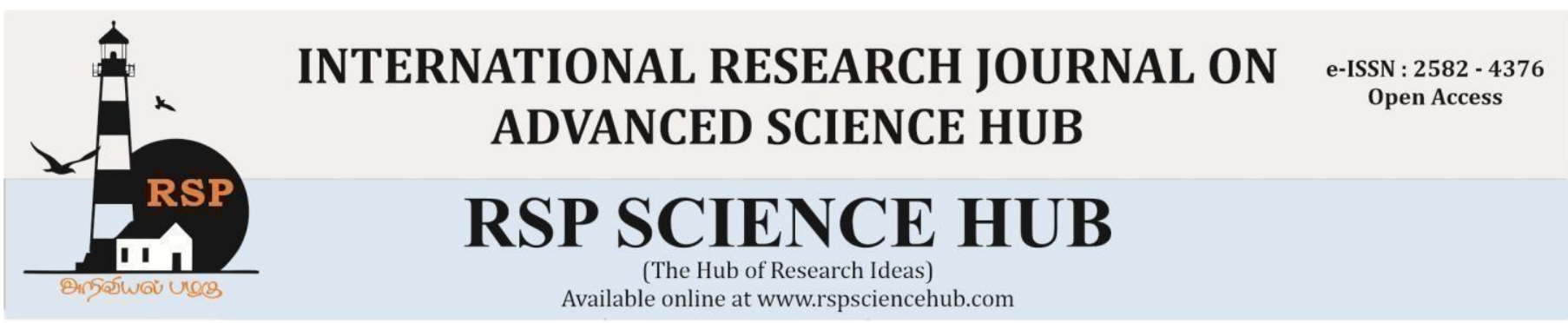

Special Issue of First International Conference on Advancements in Management, Engineering and Technology (ICAMET 2020)

\title{
Challenges for RFID Technology used in Libraries
}

Dr. Geetanjali Rana Kanwar ${ }^{1}$

${ }^{1}$ Assistant Librarian, University Library, G.B. Pant University of Agriculture and Technology, Pantnagar,

Uttarakhand, India.

geetanjali.rk@gmail.com ${ }^{1}$

Abstract

The technological advancements has taken fast pace globally throughout the world during last 10 years. The more and more digital impact has been seen in different areas related to every field. Private as well as government sectors put large emphasis and financial budget involvements to make most of the activities digital among people. The new RFID technology developed and when implemented in the libraries, it reduces the workload of circulating books through digital process of check-in and checkout among the users. RFID reduces time required to perform the Circulation operations in the libraries as compared to manual activities. RFID tags are capable and much faster than barcodes and with the help of handheld reader; items in the stack can be read at the same time. Apart from the advantages, the technology RFID has to face many challenges for its implementation and smooth working in the digital environment. The financial budget involved in it is very high. Due to chances of tag removal and tag collision, it needs extra care to be given. RFID is an excellent advanced technology but it needs to improve security factors and standards of its products and services.

Keywords: RFID, RFID Tagged, Barcode, RFID Technology, Automation, Collision, Security, Standardization.

\section{Introduction}

The advancement in technologies has changed the world of libraries and their set up in the digital environment. The libraries have now turned to digital libraries and varied software's and hardware's are implemented to run the activities. RFID is a new technology that emerged for the libraries for automation. It is used for object identification and tracking. It can hold more data than barcode technology and has ability to change the stored data.

The system of RFID needs special software to integrate with database management of library available in the form of in-house used software. When borrowing a book with the aid of Smart Cards / ID, the patron using the RFID device displays a screen display option for self-check-out. A passbook id/ code, any identification number, or any unique identity code that is already listed in the database installed under the RFID system must be recognized by the patron. The built-in RFID reader of the device recognizes the books that the patron chooses. RFID readers read the RFID tag on a book and confirm the self-check-in / out of a book.

\section{Application in RFID Library Management System}

\subsection{Book Drops}

Anywhere, inside or outside the library, the Book Drops may be found. In remote locations outside the library, it can be changed. It is $24 \mathrm{X} 7$ hours and the library items can be returned at any time, except when the library is closed, due to its versatility and Convenience. 


\subsection{RFID Transponder or Tagging}

It has the storage potential to rewrite again without touch or line of sight conditions relevant to the particular object to which they are attached. The details in the tag contains information regarding an item 's identity, evidence of ownership, the original place of storage, the status of the loan and the history of the transactions made by the employer. As per the library collection, which includes library media, books, CDs, DVDs and tapes, RFID tags have been specifically designed.

\subsection{Counter Station}

It is a staff-assisted station for library tasks such as credit, return, marking, sorting, etc. It has module arming / disarming, module tagging and module sorting. The Arming / Disarming module makes it possible to set / reset the Electronic Article Surveillance (EAS) bit within the library content tag to activate / not activate the EAS gate alarm.

\subsection{Patron self-check-in /outstation}

It functions as a touch screen computer and a built-in RFID scanner, along with special personal recognition, book and other media handling and circulation applications. It reacts according to the data on the database and server that is open. The patron is known by a library ID card, barcode card, or personal ID number (PIN) and is asked to pick one or more books on a screen to check out the next action. The patron positions the book(s) in front of him after selecting the checkout.

\subsection{Shelf Management}

2.6.

It is a handheld reader and a base station that is regarded as a form of portable scanner. It has three key specifications:

- Inventory inspection of the entire stock of library

- Search for requested individual books

- Check for miss-helved books

\subsection{Anti-theft Detection}

RFID has EAS Gates, which serves as the library RFID management system's anti-theft component that detects the same RFID tags embedded in the library objects. Each layer present in the RFID tag is capable of tracking objects of about 1 meter and when a borrowed object passed through the security gates, it will activate the alarm system. As the client passes through with a borrowed library stuff, the alarm will sound and lights on the gate will flash. The security gates also indicate the number of individuals entering. [1-4]

\section{Essential Features of RFID in Library}

The library RFID system consists of RFID tags, a self- check-in / out station, a self-return system / book drop system, a tagging / programming station, safety gate / s, an inventory / digital library assistant shelf scanner, conveyor belts and staff workstation sorting systems, etc.

\subsection{RFID Tags}

The RFID tag is a small radio device such as a transponder, smart tag, smart mark, or barcode for the radio. It has an integrated circuit or small silicon chip that contains a specific identification number (ID). It has an antenna consisting of a smooth, metallic conductive coil and a chip of less than half a millimeter that sends and receives radio waves. The tag is flexible, thin paper and approximately 2 "x 2 "in size, allowing it to be put in the collection of a library on the inside cover of each book. It has an attached antenna and a small chip that stores bibliographical data including a unique ID number to identify each object protected by the parent organization's logo sticker.

\subsection{Smartcards}

Patrons who are specially built with a special machine-readable barcode as the identification key use smart cards. They are tagged in the library management system database and when the patron places this card on the self-check-in / out kiosk, the book transaction account related to them is opened.

\subsection{Readers and Antenna}

The RFID interface consists of a reader. The reader 
units are transceivers that are a transmitter and receiver combination. The role is to search for a tag and receive information from it. The RFID reader translates RFID tags into radio waves. These radio waves are converted into a shape that can be transmitted to software that is middleware. The RFID reader uses antennas that can read information stored in the RFID tag to communicate with the RFID chip. It also updates the latest data with it. RFID reader receives commands the connectivity from the application software and communicates with tags.

\subsection{Middleware}

The implementation of the RFID framework includes middleware and software applications. The flow of information between readers and the backend is co-operated by middleware as the mediator. It helps extract information from RFID tags and control the flow of data to the backend. It performs various functions, such as integration of readers, fundamental filtering, and power. RFID middleware retrieves reader data, philtres application software data feeds, reader network output, generates stock, monitoring tag, capturing history and analyzing tag-read events for proper application and optimization.

\subsection{Server}

A server is equipped with an RFID system that offers full database information accessible via the library. It is a bridge between the various components of communication. It collects information from one or more readers and reviews information against its own database or shares information with the integrated library management system's circulation database. With the transaction details available in the server database, reports can be produced. Back up from the series from the server ensures that RFID system is working properly.

\subsection{Self-check-in/outstation}

The Self-Check-out station is a touch-screen device with a built-in RFID scanner, personal identification software, paper handling and circulation. Without any help from library personnel, patrons can search the documents themselves by following the touch screen menu.

\subsection{Book-Drop (Return Station)}

Book-Drop (Return Station): The book drop system consists of a screen book drop and a printer receipt. It helps patrons to return library documents automatically. A reader placed in a book drop enables the RFID tags to be read as the patron drops the papers. This removes conventional manual library operation by checking-in and deactivating the library staff's security protection. It automatically checks-in the text, removes it from the library account of the patron and reactivates the security feature. It absolutely locks down the patron's account after completing the transaction within a fewseconds.

\subsection{Security Gates}

Safe gate/ EAS (Electronic Article Surveillance) is a library-used anti-theft device. It plays an important role in recognizing a library paper that is not borrowed or illegally checked-out. Theft detection is an integral feature of the chip that performs both the identification of the object and the antitheft role within the RFIDtag.

\subsection{Automated sorting station}

For sorting stations, several bins or re-shelving areas are automated to take books from the return station to check them in. By deciding their shelving position in less time and less workers involved, the device confirms that the books are re-shelved. In the automated sorting station, book drop readers are used to retrieve books from the readers by adding them.

\subsection{Staff work Station}

The staff workstation is a staff-assisted station used in a library to load and unload documents, programmed new documents, sort documents, etc. It consists of a personal computer and a reader. The accession number of the document is read with the aid of the barcode scanner during the programming / tagging of a new library document / book on the reader, and then the data is downloaded from the library management system. With the support of readers, tagging is finished.

\subsection{Inventory Control}

With a portable handheld scanner, inventory and shelf reading can be carried out. The reader transmits the server's identification number, which in turn sends it 
and returns the response in real time. Information can be downloaded from the database available on the handheld reader using library management software for inventory control. The shelf management system makes it easier for library employees to find the documents on the shelves and classify them.

\section{Structural Parts of RFID System}

- Electronically configured RFID tags / transponders with specific details

- Unit for Self Review

- Return of External Book

- Staff and Station for Conversion

- Sensors or Readers to question tags.

- Aerial Antenna.

- The server on which the software that interfaces with the software of the integrated library is loaded.

- Printer with RFID labels

- Handheld Reader

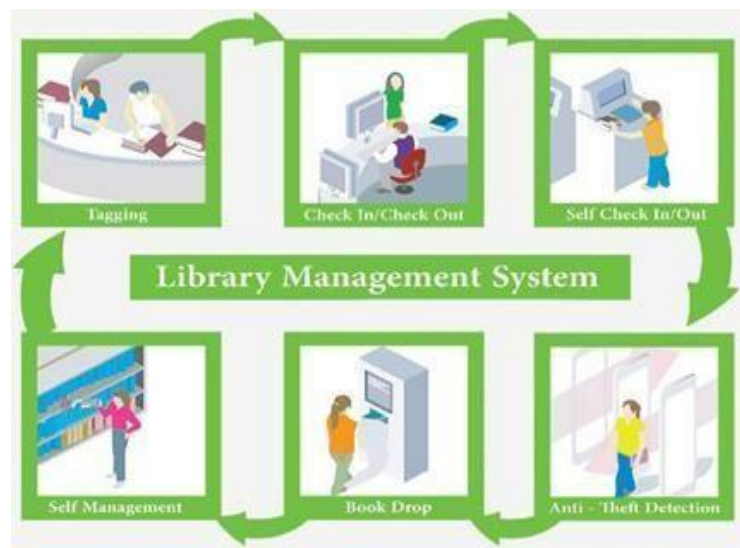

Fig.1- Structural Parts of RFID System

\section{Working of RFID System}

RFID technology operates by means of paperthin RFID tags inserted within each document's cover. The Library Management Program already provides full information about each related information when a user brings a document for issue / return and transmits the details to the software and the document is released in a few seconds without any assistance from the library staff. The antenna at the exit security gates automatically reads the information present on RFID tag to verify whether the material going outside the library is properly issued or not. In case, it is not issued, a bugler alarm starts on security gates. RFID technology is also used for stock taking purpose with the help of handheld reader. A schematic diagram in figure-2 shows system of RFID system inlibrary.[5-10]

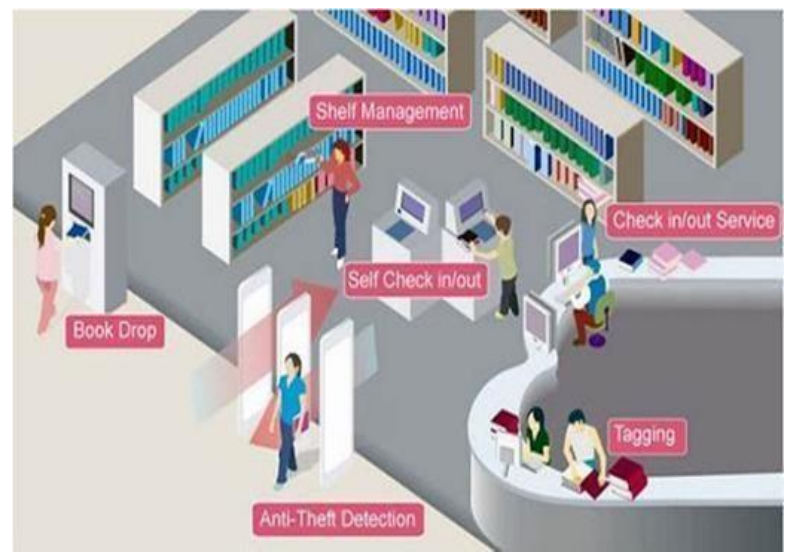

Figure-2- Working Structure of RFID System

Within the books, RFID tags are inserted and an alarm at the security gates near the exit doors is mounted. Up to $2 \mathrm{~KB}$ of RFID, tags will store data. It is possible to repair the RFID tag within the back cover of a book or directly on CDs and images. This tag is fitted with a chip and an antenna that is programmable. An engraved antenna and a microchip with a capacity of at least 64 bits are included in each thin paper tag. There are three kinds of 'read only',' WORM', and 'read/write' marks. Tags can only read if the identification is encoded at the time of manufacture and not rewritable. WORM '(write many once read) tags are configured by the company, but can modify or add details without the option to rewrite them later' Read/Write tags 'that are selected by most libraries. It is normal to have part of the read / write tag protected against rewriting in libraries using RFID, such as the item's identification number. The signal is identified by a receiver system called a reader as it reaches its radio range and decodes the number for analysis. The RFID device reader interrogates the tags and provides the reading output 
www.rspsciencehub.com

to enable immediate data capture when passed alongside the objects. The devices used inside the building are called 'readers' while the devices used at the exits of the building are called 'sensors'. To help process the identification of the objects and activate / deactivate the tag antitheft feature, an antenna is linked to the reader. In the case of larger objects, additional antenna addition will increase the amount of item processed.

Full database records and backups are available on the server involved in the RFID system. It is the gateway of communication for the various components of the RFID device involved. The data is obtained from one or more of the readers and information is shared with the circulation database. Its software includes the SIP / SIP2 (session initiation protocol) layer, the NCIP or SLNP APIs (Application Programming Interface) required to interface it with the software of the integrated library.

The RFID Label Printer is used to print the labels on the system for software installations. Without touching them, you can pass the handheld reader along with the items on the shelves. It is used in stock verification, used in book-miss-shelved search, on request search for individual books. The Shelf Check Device detects users with an RFID-ID card. In front of the self-check device, users can place objects on the reader surface to be registered under the name of the individual user. Multiple items can be checked out at the same time.

Externally open in libraries, Book Drop Station provides automated services to return books when the library is closed. It is a computer with a slot integrated into a wall with an RFID Reader chip. With the help of Smart Cards / ID, the user recognizes the patron and then puts the books into the slot. Upon completion of the return, the patron receives an automated print receipt generated by the machine showing how many and which books are returned by the library management database available and integrated. Staff and Conversion Station consist of an antenna, electronic module and power supply. The additional software windows Integrated into library management Systems.
Volume 02 Issue 10S October 2020

6. Some Indian Libraries using RFID Technology

- Indian Law University (NewDelhi)

- Indian Institute of Management(Shillong)

- National Institute of Technology(Rourkela)

- NASSDOC (New Delhi)

- National Center for Biologicalscience (Bangalore)

- National Chemical Laboratory(Pune)

- National Institute of Technology(Surat)

- Parliament library (NewDelhi)

- Punjab University(Chandigarh)

- Punjabi University(Patiala)

- Ram Manohar Lohiya Law University (Lucknow)

- University of Jammu(Jammu)

- University of Kashmir(Srinagar),

- University of Pune(Pune)

- Dayanand Sagar College ofEngineering (Bangalore)

- DESIDOC (New Delhi), Gautam Buddha University (GraterNoida)

- Indian Institute of Management(Lucknow)

- Indian Institute of Technology(Madras)

- Indian Institute of Technology(Kharagpur)

- Indian Institute of Science(Bangalore)

\section{Challenges of RFID Technology}

When applied to the libraries, RFID technology has several problems. In libraries, the main challenges of RFID technology are:

- RFID systems are costly compared to barcode systems.

- RFID technology is hard to grasp.

- Debatably less precis 
- RFID tags are bigger than labels of barcodes.

- Tags are unique to the programme. No one tag suits all

- Possibility of unauthorized reading of cards from patrons

- Chances of answering at the same time with more than one tag

\section{Drawbacks of RFID Technology}

\subsection{Dead areas and orientation problems}

Often the RFID device faces the issue of having a weaker signal and low read speeds, causing a problem when the tag is rotated into an orientation that does not match well with the reader.

\subsection{Security concerns}

RFID is not a bar-coding line-of - sight technology, new security problems could arise. For high-security activities such as payment methods, RFID is used, and cheating is often a possibility.

\subsection{Tough-to-grasp RFID technology}

RFID includes knowledge of the various tags and frequencies as well as how RFID equipment can be used. To run the entire system, workers involved in the management of the RFID system need appropriate training and skills.

\subsection{Ghost tags}

The issue of reading several tags simultaneously may cause the reader to read a tag that does not exist. There is some kind of read verification, CRC, which should be enforced in the tag, the reader or the tag read data.

\subsection{Vulnerable to damage}

The tags can be impaired by water, static discharge, or high-powered magnetic surges such as plastics can be affected by it. When objects (like lightning strikes). Tags may be susceptible to damage caused by Electro- Static

Discharge (ESD). Tags attached to insulating materials brush against each other and build up an electro-static charge, the problem can be caused by friction. During production, refining, transport and handling, it can occur. There should never be tension on microchips.

\subsection{Unread tags}

The condition arises when reading several tags at the same time when certain tags cannot be read and there is no sure way to determine this when the items are not in sight. For barcodes, this issue will not arise, because when the barcode is scanned, it is automatically checked when a beep is read from the scanner and if it does not scan, the data can then be entered manually.

There are two types of RFID's:

- Passive RFID

- Active RFID

\subsubsection{Drawbacks of Passive RFID}

- Cannot cover whole warehouse: The reading range of passive RFID is very limited, and a mobile RFID reader needs to cover all corners. To cover all the spaces requires high costs.

- Setting up and needing software updates is too complex: RFID readers need costly infrastructure setup to read in-transit tags. Read-range is still an issue for all the involved databases to be secured. There is a need for specific readers, network connectivity, routers, etc. Software updates required by RFID for proper functioning.

- Inability to provide real-time location: Readers do not naturally come with GPS and GSM triangulating capabilities.

\subsubsection{Drawbacks of Active FID}

Due to its proprietary design,

- Failure to scale: Tags only interacted with their specified RFID readers because of proprietary protocols. 
Special readers and tags for the application are required and cannot be open-sourced.

- Prohibitive cost: Due to the limited number of tags generated and protocols, economies of scale have not been achieved, making each active RFID tag more costly. Use-cases, such as last-mile delivery, it may not be preferable where a disposable form of tag is required.

- Inability to provide real-time location due to power consumption: Readers need realtime location of the package in- transit for cellular connectivity and GPS chipsets. It means that if real-time in-transit monitoring capabilities are to be constructed, the solution becomes a power- hog.

- Complexity in setting up: Reader installations, network construction, and cabling take several hours of work to set up and maintain. Reader efficiency makes a difference in terms of RF noise and density.

\subsection{Environmental Limitations}

If a tag directly touches something metallic, it might run into problems. RFID chip timing with passive UHF tags is provided, but it acts as a chip sticking directly on a sweaty runner that changes its properties as a receiver drastically and decreases read speeds.

\subsection{Government Limitations}

The reader cannot be able to read in all bands, depending on the area and internet restrictions. Regulatory agencies that monitor which bands can be used for various purposes are available.

\subsection{Software Limitations}

Excessive information makes it impossible for the programme to store and read. A retail store that can produce millions of reads per day without filtering and reading as quickly as possible. There are also licensing arrangements for certain software solutions and they can be costly, which can block implementation.

\subsection{RFID Reader Collision}

The collision with the reader happens when the signals from two or more readers overlap. The simultaneous query tag is unable to respond, and to avoid this issue, systems must be carefully set up; many systems use an anti-collision protocol. Anticollision protocols allow the tags to be transmitted to a reader in turn. By its collision-avoidance behavior, an unauthorized reader will be able to determine the identity of the tag simply because this protocol is globally special and hard coded into the tag. This information is embedded at the time of manufacture and after learning the collision-avoidance; protocol and adversary can possibly collect more data from the tag. Back door to the tag is unchangeable.

The safety bit on read / write Molnar and Wagner describe tags as vulnerable to vandalism. It is likely that a vandal to read unchecked, and lock the bit in this location may modify this bit. The vandal could secure it with a password of its own after locking the bit. If this object were ever to enter a library, because of the password, the bit could not be changed and the tag would have to be replaced. The management is described as weak from a security point of view. The passwords are used in the library for the tag and reader to communicate, and can be intercepted by an eavesdropper. Library professionals using RFID technology come across reader and tag collisions. Tag collision raises issues with readers in reading an abundance of tags at one time. It happens when more than one tag reflects a signal, and it confuses the reader.

\subsection{RFID tags are difficult to remove}

It is difficult to remove RFID tags; some of them are very small, less than half a square millimeter, and as thin as a sheet of paper, and others can be concealed or inserted within a product that cannot be seen. They can easily be destroyed if they are removed. New Technologies make it possible to "print" RFID tags right on a product and may not at all be reversible.

\subsection{Susceptibility of tags}

RFID tags function because of radio waves, stopping the whole system from functioning effectively by blocking these radio waves. The metallic ink on book covers can also influence the propagation of radio waves. Tags may be prone to elimination as well. Most 
www.rspsciencehub.com

tags are stuck to the inside of the back cover and those who want it can remove the tag. Although not all tags are so versatile, tags may be inserted into the spines of books and this does not fix the problem of tags on CDs or DVDs. Tags may cause interfaces to each other when placed within $1 / 8$ th of inch proximity.

\subsection{Privacy}

RFID operates within a system's scope and it does not operate outside the system. No patron information is found in the tags on the books, and the relation between the patron and the object is only preserved in the secure library system and this relation is broken as soon as the book is returned. Only at a distance of a few feet can RFID tags used in libraries be read. It is also very necessary that libraries follow standard guidelines to safeguard user privacy and store no personal details on RFID tags.

\subsection{Reduction of staff duties}

It is likely that a reduction in employees can follow with the automation of many employee tasks, as the RFID system may perform various conventional manual library activities on its own if it works properly. It cannot build jobs as a professional among youths. The interest lags behind and many of the professionals turn to other possibilities for jobs.

\subsection{Trouble with metals and liquids}

RFID has had a tough relationship between liquids and metals for a long time, as both find it harder to get proper asset readings. With metal, the issue stems from the bouncing radio waves all over the place. Liquids are toxic to RFID, which can absorb signals sent from RFID.

\subsection{Debatably less reliable}

RF carries data between the tag and the reader. It provides the power of RFID, but it will not work if the RF signal strength is weak. By using more than one reader and circularly polarized or multi-axis antennas, RF dead spots need to be minimized. RF is invisible; you cannot see the results. If the RF signal is small, the presence of the tag cannot be seen. The use
Volume 02 Issue 10S October 2020

of RFID at a lower frequency will increase light and shade patterns. In addition to ISO 15693, ISO 180003 and other globs, RFID tags that comply with ISO28560.

\subsection{RFID Tags larger than Barcodes}

The size of the RFID tag is determined by its antenna size. A tag size with a higher RF should be small. The processor chip in the active tag is small compared to the antenna. The chip is a small blob and the antenna is the larger printed copper conductor that surrounds it. The larger the frequency, the smaller the antenna becomes. Therefore, higher frequencies need a small tag. Tags are unique to the submission, and one tag does not suit all of them.(6-8)

\section{Limitations of RFID Technology}

- No RFID standard has yet been set. (The AutoID Center has collaborated with the Universal Code Council and EAN International standard bodies to create the electronic product code, but it is not yet considered a standard).

- The demand should push the price down as well.

- Smart tag technology has yet to be mastered; today 20 percent of tags on average do not work properly.

- RFID readers are also suffering when reading liquids or metals.

- Accurate read rates on some products can be very accurate.

- RFID requires installing a computerized chip and inserting it, which is costly.

- The collision with the reader occurs when two signals from separate readers overlap and both are unable to respond to the tag.

- RFID has two different chips that cannot be read by the same computer, read only and readable / writable(9-10).

\section{Conclusion}

Libraries are often early technology adopters, and they have begun to use RFID technology to provide more 
www.rspsciencehub.com

effective and efficient circulation facilities and to protect library collections. Its cost factor, non-availability of standards and user privacy are the major challenges of RFID technology adoption by more libraries. The non- availability of standards, those libraries that are planning to implement RFID must use RFID tags that are ISO 28560 compliant in addition to ISO 15693, ISO 18000-3 and other global standards and protocols given by NISO. It is also very important that to protect user privacy, libraries should follow standard guidelines and store no personal information on RFID tags. RFID also deploy many young library professionals and the interest towards the profession can lag behind among youths. The problem of anti-collision and overlapping in tags can affect the efficiency of RFID system if it not works properly and provide correct results of data consumed through system. It struggles through liquid or metals and can affect the security of security gate systems under RFID. It requires upgradation and upgradation of database involved with it for proper functioning that increases its cost up to higher level during maintenance. RFID proper strong standards have not yet been introduced globally. RFID technology still needs some improvements to overcome the challenges.

\section{References}

[1].Howard, L. \& Anderson, M. (2007). RFID technology in the library environment. Georgia Library Quarterly. 44(6).

[2]. Want, R. (2006). An introduction to RFID technology. IEEE Pervasive Computer, 5(1), 25-33.

[3].Christian, Kern (2004). Radio- frequency- identification for security and media circulation in libraries. The Electronic Library, 22(4), 317-324.

[4].Ayre Lori Bowen (2006). RFID and libraries. The whole Library handbook: current data professional advice and curiosa about libraries and library services. Chicago: American Library Association.
$452-456$.

[5].Vasishta Seema, "Roadmap for RFID Implementation in Central library, PEC University of Technology". Paper presented in the International Conference on Academic Libraries, Delhi. 2009. Retrieved fromhttp://eprints.rclis.org/17693/1/ical49_196_414_1_RV.pdf

[6].Anuragi, M. (2014). RFID technology for libraries: An Indian scenario. International Journal for Research in Applied Science and Engineering and Technology, 2(11), 60-65. Retrieved from http://www.ijraset.com/fileserve.php?FID=192

[7].Albert, B., Jeng, Li-Chung Chang, Hahn-Ming, Lee, Te-En, Wei and Szu-Yu, Lin,"How to solve collision and authentication issues using RFID protocol technology". Machine Learning and Cybernetics (ICMLC) 2011. International Conference. Vol.1.2011. pp. 454-460.

[8].Isabel, Expósito and Inigo, Cuiñas (2013): Exploring the Limitations on RFID Technology in Traceability Systems at Beverage Factories". International Journal of Antenna and Propagation. vol. $1 . \quad$ Article ID 916526.pp.1.https://doi.org/10.1155/2013/916526

[9].Susy, d'Hont (2002). The cutting edge of RFID Technology and Applications for manufacturing and Distribution. Texas Instrument TIRIS.

[10].Roy, Want (2006). An Introduction to RFID Technology. IEEE Pervasive Computing. 5(1), Jan.-Mar, 25-33. 